\title{
Automorphism group of the balanced hypercube*
}

\author{
Jin-Xin Zhou ${ }^{\dagger}$, Jin Ho Kwak \\ Yan-Quan Feng, Zhen-Lin Wu \\ Mathematics, Beijing Jiaotong University, Beijing 100044, P.R. China
}

Received 11 March 2015, accepted 10 March 2016, published online 12 November 2016

\begin{abstract}
Huang and Wu in [IEEE Transactions on Computers 46 (1997), pp. 484-490] introduced the balanced hypercube $B H_{n}$ as an interconnection network topology for computing systems. In this paper, we completely determine the full automorphism group of the balanced hypercube. Applying this, we first show that the $n$-dimensional balanced hypercube $B H_{n}$ is arc-transitive but not 2 -arc-transitive whenever $n \geq 2$. Then, we show that $B H_{n}$ is a lexicographic product of an $n$-valent graph $X_{n}$ and the null graph with two vertices, where $X_{n}$ is a $\mathbb{Z}_{2}^{n-1}$-regular cover of the $n$-dimensional hypercube $Q_{n}$.
\end{abstract}

Keywords: Automorphism group, balanced hypercube, Cayley graph, arc-transitive

Math. Subj. Class.: 05C25, 20B25

\section{Introduction}

The hypercube is widely known as one of the most popular interconnection networks for parallel computing systems. As a variant of the hypercube, the balanced hypercube was proposed by Huang and $\mathrm{Wu}[8]$ to enhance some properties of the hypercube. An $n$ dimensional balanced hypercube, denoted by $B H_{n}$, is defined as follows.

Definition 1.1. For $n \geq 1, B H_{n}$ has $4^{n}$ vertices, and each vertex has a unique $n$-component vector on $\{0,1,2,3\}$ for an address, also called an $n$-bit string. A vertex $\left(a_{0}, a_{1}, \ldots, a_{n-1}\right)$ is connected to the following $2 n$ vertices:

$$
\left\{\begin{array}{l}
\left(\left(a_{0}+1\right)(\bmod 4), a_{1}, \ldots, a_{i-1}, a_{i}, a_{i+1}, \ldots, a_{n-1}\right), \\
\left(\left(a_{0}-1\right)(\bmod 4), a_{1}, \ldots, a_{i-1}, a_{i}, a_{i+1}, \ldots, a_{n-1}\right),
\end{array}\right.
$$

\footnotetext{
* This work was supported by the National Natural Science Foundation of China (11271012, 11571035, 11231008) and the Fundamental Research Funds for the Central Universities (2015JBM110).

$\dagger$ Corresponding author.

E-mail addresses: jxzhou@bjtu.edu.cn (Jin-Xin Zhou), jinkwak@ postech.ac.kr (Jin Ho Kwak), yqfeng@bjtu.edu.cn (Yan-Quan Feng), 10271079@bjtu.edu.cn (Zhen-Lin Wu)
} 


$$
\left\{\begin{array}{l}
\left(\left(a_{0}+1\right)(\bmod 4), a_{1}, \ldots, a_{i-1},\left(a_{i}+(-1)^{a_{0}}\right)(\bmod 4), a_{i+1}, \ldots, a_{n-1}\right), \\
\left(\left(a_{0}-1\right)(\bmod 4), a_{1}, \ldots, a_{i-1},\left(a_{i}+(-1)^{a_{0}}\right)(\bmod 4), a_{i+1}, \ldots, a_{n-1}\right),
\end{array}\right.
$$

for $1 \leq i \leq n-1$.
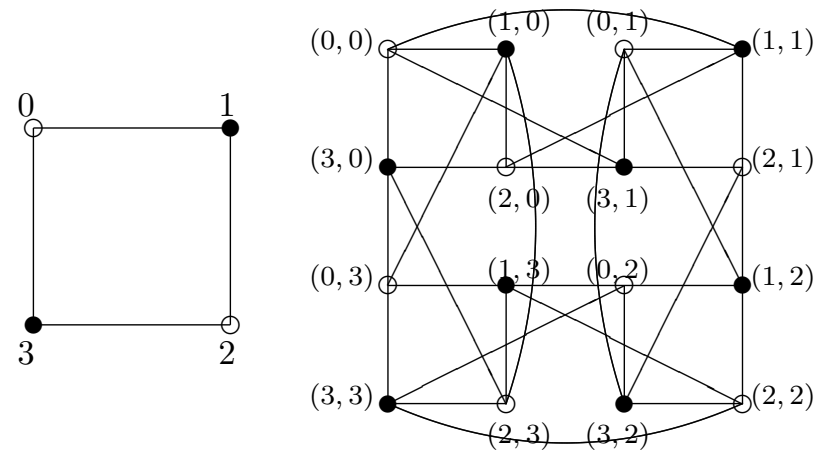

Figure 1: Two balanced hypercubes: $\mathrm{BH}_{1}$ and $\mathrm{BH}_{2}$

By now, various properties of the balanced hypercube, such as, Hamiltonian laceability, bipanconnectivity, super connectivity etc. have been extensively investigated in the literature $[7,8,9,14,16,17,18,19]$. In many situations, it is highly desired to use interconnection networks which are highly symmetric. This often simplifies the computational and routing algorithms. It has been shown that the balanced hypercube is vertex-transitive and arc-transitive (see [14, 22]). When dealing with the symmetry of graphs, the goal is to gain as much information as possible about the structure of the full automorphism groups. Recently, several publications have been put into investigation of automorphism groups of Cayley graphs having connection with interconnection networks (see, for example, $[5,10,20,21])$.

In [22], it was proved that $B H_{n}$ is an arc-transitive Cayley graph.

Definition 1.2. For $n \geq 1$, let $H_{n}$ be an abelian group defined as follows:

$$
H_{n}=\langle y\rangle \times\left\langle z_{1}\right\rangle \times\left\langle z_{2}\right\rangle \times \ldots \times\left\langle z_{n-1}\right\rangle \cong \mathbb{Z}_{2} \times \mathbb{Z}_{4} \times \mathbb{Z}_{4} \times \ldots \times \mathbb{Z}_{4} .
$$

The generalized dihedral group of $H_{n}$, denoted by $\operatorname{Dih}\left(H_{n}\right)$, is the semi-direct product of $H_{n}$ by a group $\langle x\rangle$ of order 2 with the involution $x$ inverting every element in $H_{n}$. Let $G_{n}=\operatorname{Dih}\left(H_{n}\right)=H_{n} \rtimes\langle x\rangle$ and let $S=\left\{x, x y, x z_{i}, x y z_{i} \mid i=1,2, \ldots, n-1\right\}$. Let $\Gamma_{n}$ be the following Cayley graph:

$$
\Gamma_{n}=\operatorname{Cay}\left(G_{n}, S\right) \text {. }
$$

Proposition 1.3. [22, Theorem 3.7] For each $n \geq 1, B H_{n} \cong \Gamma_{n}$ is arc-transitive.

Definition 1.4. Let $L_{n}$ be a subgroup of $H_{n}$ defined by

$$
L_{n}=\left\langle z_{1}\right\rangle \times\left\langle z_{2}\right\rangle \times \ldots \times\left\langle z_{n-1}\right\rangle \cong \underbrace{\mathbb{Z}_{4} \times \mathbb{Z}_{4} \times \ldots \times \mathbb{Z}_{4}}_{n-1} .
$$


Let $T_{n}=\operatorname{Dih}\left(L_{n}\right)=L_{n} \rtimes\langle x\rangle$. Clearly, $T_{n}$ is a subgroup of $G_{n}$ of index 2 . Set $\Omega=$ $\left\{x, x z_{i} \mid i=1,2, \ldots, n-1\right\}$, and define $X_{n}$ as the following Cayley graph:

$$
X_{n}=\operatorname{Cay}\left(T_{n}, \Omega\right) .
$$

For convenience, in what follows we shall always let $\Gamma_{n}=B H_{n}$. In [3], the authors proved the following result.

Proposition 1.5. [3, Theorem 3.4] For each $n \geq 1, B H_{n} \cong X_{n}\left[2 K_{1}\right]$, where $X_{n}$ is defined as following:

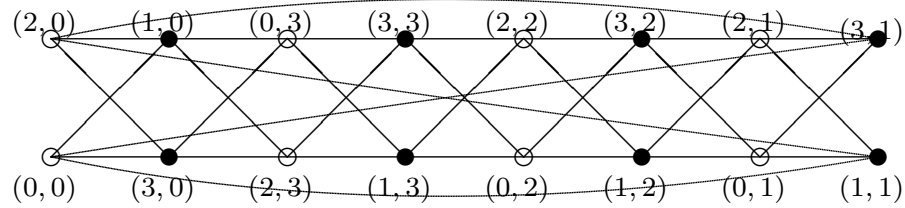

Figure 2: Another layout of $\mathrm{BH}_{2}$

By Proposition 2.1, it is easy to see that $\operatorname{Aut}\left(B H_{n}\right)=\mathbb{Z}_{2}$ 々 Aut $\left(X_{n}\right) *$. So, to determine the automorphism group of $B H_{n}$, the key is to determine the automorphism group of $X_{n}$. In this paper, we prove that $X_{n}$ is a 2-arc-transitive normal Cayley graph, and $\operatorname{Aut}\left(X_{n}\right)=$ $R\left(T_{n}\right) \rtimes \operatorname{Aut}\left(T_{n}, \Omega\right) \cong T_{n} \rtimes S_{n}$.

As the automorphism group of $B H_{n}$ is known, one may ask: Does $B H_{n}$ have a stronger symmetry property? In this paper, we show that $B H_{n}$ is arc-transitive but not 2-arc-transitive.

As another application, we prove that $X_{n}$ is a $\mathbb{Z}_{2}^{n-1}$-regular cover of the hypercube $Q_{n}$. This, together with the fact $B H_{n} \cong X_{n}\left[2 K_{1}\right]$, gives a theoretical explanation of the relationship between $B H_{n}$ and $Q_{n}$.

\section{Preliminaries}

In this section, we shall introduce some notations, terminology and preliminary results. Throughout this paper only undirected simple connected graphs without loops and multiple edges are considered. Unless stated otherwise, we follow Bondy and Murty [2] for terminology and definitions.

Let $n$ be a positive integer. Denote by $\mathbb{Z}_{n}$ the cyclic group of order $n$, by $S_{n}$ the symmetric group of degree $n$ and by $K_{n, n}$ the complete bipartite graph of order $2 n$ and valency $n$, respectively. We also use $n K_{1}, K_{n}$ and $C_{n}$ to denote the null graph, the complete graph and the cycle with $n$ vertices, respectively.

In a parallel computing system, processors are connected based on a specific interconnection network. An interconnection network is usually represented by a graph in which vertices represent processors and edges represent links between processors. Let $G$ be a simple undirected connected graph. We denote by $\operatorname{Aut}(G)$ the full automorphism group of $G$, and by $V(G)$ and $E(G)$ the sets of vertices and edges of $G$, respectively. For $u, v \in V(G)$, denote by $\{u, v\}$ the edge incident to $u$ and $v$ in $G$. For a vertex $v$ in a graph $G$, use $N_{G}(v)$ to denote the neighborhood of $v$, that is, the set of vertices adjacent to $v$.

\footnotetext{
* One can also obtain this by using [4, Theorem 5.7]. We thank a referee for pointing out this.
} 
An $s$-arc in a graph $G$ is an ordered $(s+1)$-tuple $\left(v_{0}, v_{1}, \ldots, v_{s-1}, v_{s}\right)$ of vertices of $G$ such that $v_{i-1}$ is adjacent to $v_{i}$ for $1 \leq i \leq s$ and $v_{i-1} \neq v_{i+1}$ for $1 \leq i \leq s-1$. A graph $G$ is said to be $s$-arc-transitive if $\operatorname{Aut}(G)$ is transitive on the set of $s$-arcs in $G$. In particular, 0-arc-transitive means vertex-transitive, and 1-arc-transitive means arctransitive or symmetric. A graph $G$ is edge-transitive if $\operatorname{Aut}(G)$ acts transitively on $E(G)$. Clearly, every arc-transitive graph is both edge-transitive and vertex-transitive.

\subsection{Wreath products of groups}

For a set $V$ and a group $G$ with identity element 1 , an action of $G$ on $V$ is a mapping $V \times G \rightarrow V,(v, g) \mapsto v^{g}$, such that $v^{1}=v$ and $\left(v^{g}\right)^{h}=v^{g h}$ for $v \in V$ and $g, h \in G$. The kernel of $G$ acting on $V$ is the subgroup of $G$ fixing $V$ pointwise. For two groups $K, H$, if $H$ acts on $K$ (as a set) such that $(x y)^{h}=x^{h} y^{h}$ for any $x, y \in K$ and $h \in H$, then $H$ is said to act on $K$ as a group. In this case, we use $K \rtimes H$ to denote the semi-direct product of $K$ by $H$ with respect to the action.

Let $H$ be a permutation group on a finite set $\Delta$. For convenience, let $\Delta=\{1,2, \cdots, n\}$. Let $G$ be a permutation group on a finite set $\Phi$, and let

$$
N=\underbrace{G \times G \times \cdots \times G}_{n \text { times }} .
$$

We define the action of $H$ on $N$ as following:

$$
\forall h \in H,\left(g_{1}, g_{2} \cdots, g_{n}\right)^{h}=\left(g_{1^{h^{-1}}}, g_{2^{h^{-1}}}, \cdots, g_{n^{h^{-1}}}\right), g_{i} \in G, i=1,2, \cdots, n .
$$

Then the semi-direct product of $N$ by $H$ with respect to this action is called the wreath product of $G$ and $H$, denoted by $G$ ? $H$. Clearly,

$$
G \prec H=\left\{\left(g_{1}, g_{2}, \cdots, g_{n} ; h\right) \mid g_{i} \in G, h \in H\right\} .
$$

Moreover, $G$ 々 $H$ can be viewed as a permutation group on $\Phi \times \Delta$ as following:

$$
(x, i)^{\left(g_{1}, g_{2}, \cdots, g_{n} ; h\right)}=\left(x^{g_{i}}, i^{h}\right) .
$$

Let $G$ and $H$ be two graphs. The lexicographic product $G[H]$ is defined as the graph with vertex set $V(G) \times V(H)$ and for any two vertices $\left(u_{1}, v_{1}\right),\left(u_{2}, v_{2}\right) \in V(G) \times V(H)$, they are adjacent in $G[H]$ if and only if either $u_{1}=u_{2}$ and $v_{1}$ is adjacent to $v_{2}$ in $H$, or $u_{1}$ is adjacent to $u_{2}$ in $G$. In view of [13, Theorem.], we have the following.

Proposition 2.1. [13, Theorem.] Let $X$ and $Y$ be two graphs. Then $\operatorname{Aut}(X[Y])=\operatorname{Aut}(Y)$ ? $\operatorname{Aut}(X)$ if and only if

(1) if there are two distinct vertices $u, v \in V(X)$ such that $N_{X}(u)=N_{X}(v)$, then $Y$ is connected;

(2) if there are two distinct vertices $u, v \in V(X)$ such that $N_{X}(u) \cup\{u\}=N_{X}(v) \cup\{v\}$, then the complement $\bar{Y}$ of $Y$ is connected.

\subsection{Cayley graphs}

Let $G$ be a permutation group on a set $\Omega$ and $\alpha \in \Omega$. Denote by $G_{\alpha}$ the stabilizer of $\alpha$ in $G$, that is, the subgroup of $G$ fixing the point $\alpha$. We say that $G$ is semiregular on $\Omega$ if 
$G_{\alpha}=1$ for every $\alpha \in \Omega$ and regular if $G$ is transitive and semiregular. Given a finite group $G$ and an inverse closed subset $S \subseteq G \backslash\{1\}$, the Cayley graph $\operatorname{Cay}(G, S)$ on $G$ with respect to $S$ is defined to have vertex set $G$ and edge set $\{\{g, s g\} \mid g \in G, s \in S\}$. A Cayley graph $\operatorname{Cay}(G, S)$ is connected if and only if $S$ generates $G$. Given a $g \in G$, define the permutation $R(g)$ on $G$ by $x \mapsto x g, x \in G$. Then $R(G)=\{R(g) \mid g \in G\}$, called the right regular representation of $G$, is a permutation group isomorphic to $G$. It is well-known that $R(G) \leq \operatorname{Aut}(\operatorname{Cay}(G, S))$. So, $\operatorname{Cay}(G, S)$ is vertex-transitive. In general, a vertex-transitive graph $X$ is isomorphic to a Cayley graph on a group $G$ if and only if its automorphism group has a subgroup isomorphic to $G$, acting regularly on the vertex set of $X$ (see [1, Lemma 16.3]).

For two inverse closed subsets $S$ and $T$ of a group $G$ not containing the identity 1 , if there is an $\alpha \in \operatorname{Aut}(G)$ such that $S^{\alpha}=T$ then $S$ and $T$ are said to be equivalent, denoted by $S \equiv T$. The following proposition is easy to obtain.

Proposition 2.2. If $S$ and $T$ are equivalent then $\operatorname{Cay}(G, S) \cong \operatorname{Cay}(G, T)$.

A Cayley graph $\operatorname{Cay}(G, S)$ is said to be normal if $R(G)$ is normal in $\operatorname{Aut}(\operatorname{Cay}(G, S))$ (see [15]). Let Cay $(G, S)$ be a Cayley graph on a group $G$ with respect to a subset $S$ of $G$. Set $A=\operatorname{Aut}(\operatorname{Cay}(G, S))$ and $\operatorname{Aut}(G, S)=\left\{\alpha \in \operatorname{Aut}(G) \mid S^{\alpha}=S\right\}$.

Proposition 2.3. [15, Proposition 1.5] The Cayley graph $\mathrm{Cay}(G, S)$ is normal if and only if $A_{1}=\operatorname{Aut}(G, S)$, where $A_{1}$ is the stabilizer of the identity 1 of $G$ in $A$.

\subsection{Covers of graphs}

An important tool in studying symmetry properties of graphs is the covering technique. An epimorphism $\wp: \widetilde{X} \rightarrow X$ of graphs is called a regular covering projection if there is a semiregular subgroup $\mathrm{CT}(\wp)$ of the automorphism group $\operatorname{Aut}(\widetilde{X})$ of $\tilde{X}$ whose orbits in $V(\widetilde{X})$ coincide with the vertex fibers $\wp^{-1}(v), v \in V(X)$, and the arc and edge orbits of $\mathrm{CT}(\wp)$ coincide with the arc fibers $\wp^{-1}((u, v)), u \sim v$, and the edge fibers $\wp^{-1}(\{u, v\})$, $u \sim v$, respectively. In particular, we call the graph $\widetilde{X}$ a regular cover of the graph $X$. The semiregular group CT $(\wp)$ is the covering transformation group. If CT $(\wp)$ is isomorphic to an abstract group $N$ then we speak of $\widetilde{X}$ as a regular $N$-cover of $X$. For more results on the covering of graphs, we refer the reader to [6, 12].

Let $X$ be a connected $k$-valent graph and let $G \leq \operatorname{Aut}(X)$ act transitively on the 2-arcs of $X$. Let $N$ be a normal subgroup of $G$. The quotient graph $X_{N}$ of $X$ relative to $N$ is defined as the graph with vertices the orbits of $N$ in $V(X)$ and with two orbits adjacent if there is an edge in $X$ between those two orbits. In view of [11, Theorem 9], we have the following.

Proposition 2.4. If $N$ has more than two orbits in $V(X)$, then $N$ is semiregular on $V(X)$, $X_{N}$ is a $k$-valent graph with $G / N$ as a 2-arc-transitive group of automorphisms, and $X$ is a regular $N$-cover of $X_{N}$.

\section{Automorphism group of the balanced hypercube}

In this section, we shall determine the full automorphism group of the balanced hypercube. From Proposition 1.5 we know that $\Gamma_{n} \cong X_{n}\left[2 K_{1}\right]$, and by Proposition $2.1, \operatorname{Aut}\left(\Gamma_{n}\right) \cong$ $\mathbb{Z}_{2}$ ? Aut $\left(X_{n}\right)$. So, the key step is to determine the automorphism group of $X_{n}$. 
Lemma 3.1. For $n \geq 1, X_{n}$ is a 2-arc-transitive normal Cayley graph, and furthermore, $\operatorname{Aut}\left(X_{n}\right)=R\left(T_{n}\right) \rtimes \operatorname{Aut}\left(T_{n}, \Omega\right)$, where $R\left(T_{n}\right) \cong T_{n}=\operatorname{Dih}\left(\mathbb{Z}_{4}^{n-1}\right)$ and $\operatorname{Aut}\left(T_{n}, \Omega\right) \cong$ $S_{n}$.

Proof. Clearly, $X_{1} \cong K_{2}$ and $X_{2} \cong C_{8}$. It is easy to see that the statement is true for these two cases. In what follows, assume that $n \geq 3$. We first prove the following two claims.

Claim $1 \operatorname{Aut}\left(T_{n}, \Omega\right) \cong S_{n}$.

Since $\Omega$ generates $T_{n}$, Aut $\left(T_{n}, \Omega\right)$ acts faithfully on $\Omega$, and hence $\operatorname{Aut}\left(T_{n}, \Omega\right) \leq S_{n}$.

It is easy to verify that $x z_{1}, z_{1}^{-1} z_{i}(2 \leq i \leq n-1), z_{1}^{-1}$ generate $T_{n}$ and they satisfy the same relations as $x, z_{i}(1 \leq i \leq n-2), z_{n-1}$. This implies that the map

$$
\alpha: x \mapsto x z_{1}, z_{i} \mapsto z_{1}^{-1} z_{i+1}(1 \leq i \leq n-2), z_{n-1} \mapsto z_{1}^{-1},
$$

induces an automorphism of $T_{n}$. Clearly, for each $1 \leq i \leq n-2,\left(x z_{i}\right)^{\alpha}=x z_{i+1}$, and $x \mapsto x z_{1}$ and $\left(x z_{n-1}\right)^{\alpha}=x$. This implies that $\alpha$ cyclicly permutates the elements in $\Omega$, and so $\alpha \in \operatorname{Aut}\left(T_{n}, \Omega\right)$.

Similarly, for each $2 \leq i \leq n-1$, we define a map as the following:

$$
\beta_{i}: x \mapsto x, z_{1} \mapsto z_{i}, z_{i} \mapsto z_{1}, z_{j} \mapsto z_{j}(1 \leq i, j \leq n-1 \text { and } i \neq j)
$$

Then $\beta_{i}$ induces an automorphism of $T_{n}$, and furthermore, $\beta_{i}$ interchanges $x z_{1}$ and $x z_{i}$ and fixes all other elements in $\Omega$. Hence, $\beta_{i} \in \operatorname{Aut}\left(T_{n}, \Omega\right)$ and by elementary group theory, we obtain that the subgroup generated by $\beta_{i}(2 \leq i \leq n-1)$ is isomorphic to $S_{n-1}$. Since $S_{n-1}$ is maximal in $S_{n}$, one has $\left\langle\alpha, \beta_{i} \mid 2 \leq i \leq n-1\right\rangle \cong S_{n}$. It follows that $\operatorname{Aut}\left(T_{n}, \Omega\right)=\left\langle\alpha, \beta_{i} \mid 2 \leq i \leq n-1\right\rangle \cong S_{n}$.

Claim 2 For any $x z_{i}$, there are $(n-2) 6$-cycles in $X_{n}$ passing through the 2 -arc $\left(x, 1, x z_{i}\right)$, namely, $C^{i, j}=\left(1, x, z_{j}^{-1}, x z_{i} z_{j}^{-1}, z_{j}^{-1} z_{i}, x z_{i}, 1\right)$ with $j \neq i$ and $1 \leq j \leq n-1$.

By Claim $1, \operatorname{Aut}\left(T_{n}, \Omega\right)$ acts 2 -transitively on $\Omega$. It is well-known that a vertex-transitive graph is 2-arc-transitive if and only if the vertex-stabilizer $\operatorname{Aut}\left(X_{n}\right)_{v}$ is 2-transitive on the set of vertices adjacent to $v$. So, $X_{n}$ is 2 -arc-transitive. To prove the claim, it suffices to show that the statement is true for the case when $i=1$.

First, for any $2 \leq j \leq n-1$, one may easily check that $C^{1, j}=\left(1, x, z_{j}^{-1}, x z_{1} z_{j}^{-1}\right.$, $\left.z_{1} z_{j}^{-1}, x z_{1}, 1\right)$ is a 6 -cycle passing through the 2 -arc $\left(x, 1, x z_{1}\right)$. Let $C^{\prime}$ be an arbitrary 6 -cycle passing through $\left(x, 1, x z_{1}\right)$. Then there exist $s_{1}, s_{2}, t_{1}, t_{2} \in \Omega$ such that $C^{\prime}=$ $\left(1, x, s_{1} x, s_{2} s_{1} x=t_{2} t_{1} x z_{1}, t_{1} x z_{1}, x z_{1}, 1\right)$, where $s_{1} \neq x, s_{2} \neq s_{1}, t_{1} \neq x z_{1}$ and $t_{1} \neq t_{2}$. Clearly, $s_{1}=x z_{j}$ for some $1 \leq j \leq n-1$. In the rest of the proof of Claim 2 the following well-known fact will be frequently used.

Fact Every element in $\left\langle z_{1}\right\rangle \times\left\langle z_{2}\right\rangle \times \ldots \times\left\langle z_{n-1}\right\rangle$ can be uniquely written in the following form

$$
z_{1}^{a_{1}} z_{2}^{a_{2}} \ldots z_{n-1}^{a_{n-1}}, a_{i} \in \mathbb{Z}_{4}(1 \leq i \leq n-1)
$$

If $s_{2}=x$, then $x x z_{j} x=t_{2} t_{1} x z_{1}$. It follows that $z_{j} x=t_{2} t_{1} x z_{1}$ and hence $z_{j} z_{1}=t_{2} t_{1}$. If $t_{2}=x$, then $t_{1}=x z_{k}$ for some $1 \leq k \leq n-1$, and so $z_{j} z_{1}=z_{k}$. By Fact, this is impossible. If $t_{2}=x z_{\ell}$ for some $1 \leq \ell \leq n-1$, then we have either $t_{1}=x$ or $t_{1}=x z_{p}$ for some $1 \leq p \leq n-1$. For the former, we have $z_{j} z_{1}=z_{\ell}^{-1}$, and for the latter, we have $t_{2} t_{1}=x z_{\ell} x z_{p}=z_{\ell}^{-1} z_{p}=z_{j} z_{1}$. From the above Fact, both of these cannot happen. 
If $s_{2}=x z_{i}$ for some $1 \leq i \leq n-1$, then $x z_{i} x z_{j} x=t_{2} t_{1} x z_{1}$. It follows that $z_{i}^{-1} z_{j} x=t_{2} t_{1} x z_{1}$ and hence $z_{i}^{-1} z_{j} z_{1}=t_{2} t_{1}$. If $t_{1}=x z_{k}$ and $t_{2}=x z_{p}$ for some $1 \leq k, p \leq n-1$, then $t_{2} t_{1}=z_{p}^{-1} z_{k}=z_{i}^{-1} z_{j} z_{1}$. This is also impossible. If $t_{1}=x$ and $t_{2}=x z_{p}$ for some $1 \leq p \leq n-1$, then $t_{2} t_{1}=z_{p}^{-1}=z_{i}^{-1} z_{j} z_{1}$. This is also impossible. So, we must have $t_{1}=x z_{k}$ and $t_{2}=x$ for some $1 \leq k \leq n-1$. Then $t_{2} t_{1}=z_{k}=z_{i}^{-1} z_{j} z_{1}$. Clearly, $s_{1} \neq s_{2}$. Then $z_{k}=z_{j}$ and $z_{i}=z_{1}$. That is $s_{2}=x z_{1}, t_{2}=x, t_{1}=s_{1}=x z_{j}$. It follows that $C^{\prime}=C^{1, j}=\left(1, x, z_{j}^{-1}, x z_{1} z_{j}^{-1}, z_{j}^{-1} z_{1}, x z_{1}, 1\right)$.

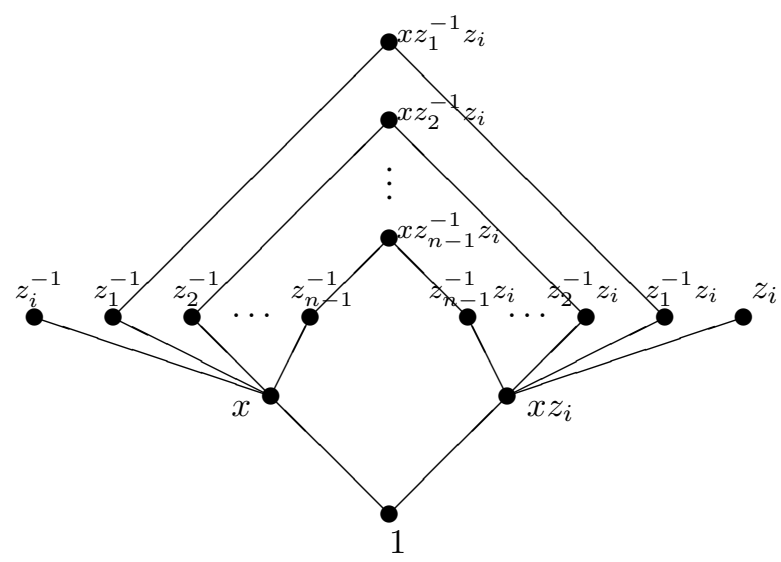

Figure 3: 6-cycles passing through $\left(x, 1, x z_{i}\right)$

Now we are ready to complete the proof. Let $A=\operatorname{Aut}\left(X_{n}\right)$ and let $A_{1}$ be the stabilizer of the identity 1 in $A$. Let $A_{1}^{*}$ be the kernel of $A_{1}$ acting on $\Omega$. Then $A_{1}^{*}$ fixes every element in $\Omega$. For any $x z_{i}(1 \leq i \leq n-1)$, by Claim 2 , there are exactly $(n-2) 6$-cycles in $X_{n}$ passing through the 2 -arc $\left(x, 1, x z_{i}\right)$, namely, $C^{i, j}=\left(1, x, z_{j}^{-1}, x z_{i} z_{j}^{-1}, z_{j}^{-1} z_{i}, x z_{i}, 1\right)$ with $j \neq i$ and $1 \leq j \leq n-1$ (see Fig. (3)). Note that the neighborhood of $x$ is $\left\{1, z_{i}^{-1} \mid 1 \leq\right.$ $i \leq n-1\}$ and the neighborhood of $x z_{i}$ is $\left\{1, z_{i}, z_{j}^{-1} z_{i} \mid 1 \leq i, j \leq n-1, j \neq i\right\}$. Since there are no 6 -cycles passing through $z_{i}^{-1}, x, 1, x z_{i}$ and $z_{i}$, it follows that $A_{1}^{*}$ fixes $z_{i}^{-1}$ and $z_{i}(1 \leq i \leq n-1)$.

By [3, Lemma 4.2], $X_{n}$ has girth 6 , and so $C^{i, j}$ is the unique 6 -cycle passing through $z_{j}^{-1}, x, 1, x z_{i}, z_{j}^{-1} z_{i}$. As $A_{1}^{*}$ fixes $z_{j}^{-1}, x, 1$ and $x z_{i}, A_{1}^{*}$ must fix $z_{j}^{-1} z_{i}$. By the arbitrariness of $i, j$, we obtain that $A_{1}^{*}$ fixes every vertex of the set $\left\{z_{i}^{-1}, z_{i}, z_{j}^{-1} z_{i} \mid 1 \leq i, j \leq n-1, j \neq\right.$ $i$ \} which is just the set of vertices at distance 2 from the identity 1 . By the vertex-transitivity and connectivity of $X_{n}, A_{1}^{*}$ fixes all vertices of $X_{n}$. It follows that $A_{1}^{*}=1$, and so $A_{1}$ acts faithfully on $\Omega$. Therefore, $A_{1} \lesssim S_{n}$. By Claim 1 , Aut $\left(T_{n}, \Omega\right) \cong S_{n}$, and since $\operatorname{Aut}\left(T_{n}, \Omega\right) \leq A_{1}$, one has $\operatorname{Aut}\left(T_{n}, \Omega\right)=A_{1}$. By Proposition 2.3, $X_{n}$ is normal, and so $A=R\left(T_{n}\right) \rtimes \operatorname{Aut}\left(T_{n}, \Omega\right)$.

Now we are ready to determine the automorphism group of $\mathrm{BH}_{n}$.

Theorem 3.2. For $n \geq 1$, Aut $\left(B H_{n}\right)=\mathbb{Z}_{2} \curlywedge\left(T_{n} \rtimes S_{n}\right)$.

Proof. By Proposition 1.5, $B H_{n} \cong X_{n}\left[2 K_{1}\right]$. By Proposition 2.1, Aut $\left(B H_{n}\right) \cong \mathbb{Z}_{2}$ 2 $\operatorname{Aut}\left(X_{n}\right)$. From Theorem 3.1 we obtain that $\operatorname{Aut}\left(X_{n}\right)=R\left(T_{n}\right) \rtimes \operatorname{Aut}\left(T_{n}, \Omega\right) \cong T_{n} \rtimes S_{n}$. It follows that $\operatorname{Aut}\left(B H_{n}\right)=\mathbb{Z}_{2} \succ\left(T_{n} \rtimes S_{n}\right)$. 


\section{Related results}

As the automorphism group of $B H_{n}$ is known, we can obtain more information about the symmetry properties of $B H_{n}$. By Proposition 1.3, $B H_{n}$ is arc-transitive, and by Theorem 3.1, $X_{n}$ is 2-arc-transitive. It is natural to ask: whether $B H_{n}$ has much stronger symmetry property? We answer this in negative by showing that $B H_{n}$ is not 2-arc-transitive.

Theorem 4.1. For $n \geq 2, B H_{n}$ is arc-transitive but not 2-arc-transitive.

Proof. Suppose, by way of contradiction, that $B H_{n}$ is 2-arc-transitive. Recall that $B H_{n}=$ $\operatorname{Cay}\left(G_{n}, S\right)$. Then the vertex-stabilizer $\operatorname{Aut}\left(B H_{n}\right)_{1}$ of the identity 1 of $G_{n}$ in $\operatorname{Aut}\left(B H_{n}\right)$ is 2-transitive on $S$. That is, for any two distinct ordered pairs from $S \times S$, say $\left(u_{1}, v_{1}\right)$ and $\left(u_{2}, v_{2}\right)$, there exists $\alpha \in \operatorname{Aut}\left(B H_{n}\right)_{1}$ such that $\left(u_{1}, v_{1}\right)^{\alpha}=\left(u_{2}, v_{2}\right)$. In particular, there exists $\alpha \in \operatorname{Aut}\left(B H_{n}\right)_{1}$ such that $(x, x y)^{\alpha}=\left(x, x z_{1}\right)$. This implies that $x$ and $x z_{1}$ have the same neighborhood because $x$ and $x y$ have the same neighborhood. However, from [22, Lemma 3.8], we see that $x y$ is the unique vertex which has the same neighborhood as $x$, a contradiction.

By Proposition $1.5, B H_{n} \cong X_{n}\left[2 K_{1}\right]$. As a consequence of Theorem 3.1, we can also prove that $X_{n}$ is a $\mathbb{Z}_{2}^{n-1}$-regular cover of the hypercube $Q_{n}$. This reveals the relationship between the balanced hypercube $B H_{n}$ and the hypercube $Q_{n}$.

Lemma 4.2. For $n \geq 1$, let $N=\mathbb{Z}_{2}^{n}$. Let $G=\operatorname{Cay}(N, S)$ be a connected $n$-valent Cayley graph. Then $G$ is isomorphic to the n-dimensional hypercube $Q_{n}$.

Proof. It is well-known that $Q_{n}$ is a Cayley graph on $N$ with respect to the subset

$$
T=\{(1,0,0, \cdots, 0),(0,1,0, \cdots, 0), \cdots,(0,0,0, \cdots, 1)\} .
$$

Viewing $N$ as an $n$-dimensional vector space on the field $\mathbb{Z}_{2}$, one may see that $T$ is a basis of $N$. Since $G$ is an $n$-valent Cayley graph, one has $|S|=n$, and since $G$ is connected, one has $N=\langle S\rangle$. This means that $S$ is also a basis of $N$. So, there is an automorphism of $N$ which maps $S$ to $T$. By Proposition 2.2, $G \cong Q_{n}$, as desired.

Theorem 4.3. For $n \geq 3, X_{n}$ is a $\mathbb{Z}_{2}^{n-1}$-regular cover of $Q_{n}$.

Proof. By Theorem 3.1, $R\left(T_{n}\right)$ is normal in $\operatorname{Aut}\left(X_{n}\right)$. Remember that $T_{n}=\operatorname{Dih}\left(L_{n}\right)=$ $L_{n} \rtimes\langle x\rangle$, where

$$
L_{n}=\left\langle z_{1}\right\rangle \times \ldots \times\left\langle z_{n-1}\right\rangle \cong \underbrace{\mathbb{Z}_{4} \times \ldots \times \mathbb{Z}_{4}}_{n-1 \text { times }},
$$

and $x$ is an involution inverting every element in $L_{n}$. Set $Z=\left\langle R\left(z_{1}^{2}\right)\right\rangle \times \ldots \times\left\langle R\left(z_{n-1}^{2}\right)\right\rangle$. Then

$$
Z \cong \underbrace{\mathbb{Z}_{2} \times \ldots \times \mathbb{Z}_{2}}_{n-1 \text { times }}
$$

and $Z$ is just the center of $R\left(T_{n}\right)$. It follows that $Z$ is characteristic in $R\left(T_{n}\right)$. Since $R\left(T_{n}\right) \unlhd \operatorname{Aut}\left(X_{n}\right)$, one has $Z \unlhd \operatorname{Aut}\left(X_{n}\right)$. Consider the quotient graph $Y_{n}$ of $X_{n}$ relative to $Z$. Clearly, $Z$ is semiregular on the vertex-set of $X_{n}$, and so it has more than 2 orbits on $V(X)$. Since $X_{n}$ is 2 -arc-transitive, by Proposition $2.4, Y_{n}$ is also an $n$-valent graph with 
$\operatorname{Aut}\left(X_{n}\right) / Z$ as a 2-arc-transitive automorphism group, and $X_{n}$ is a $Z$-regular cover of $Y_{n}$. To complete the proof, it suffices to prove that $Y_{n} \cong Q_{n}$.

Noting that $Z \unlhd R\left(T_{n}\right)$ and $R\left(T_{n}\right)$ is regular on $V\left(X_{n}\right), R\left(T_{n}\right) / Z$ is regular on $V\left(Y_{n}\right)$. It follows that $Y_{n}$ is a Cayley graph on $R\left(T_{n}\right) / Z$. As $R\left(T_{n}\right)=\operatorname{Dih}\left(L_{n}\right)$, one has $R\left(T_{n}\right) / Z \cong \mathbb{Z}_{2}^{n}$. Since $Y_{n}$ has valency $n$, by Lemma 4 .2, one has $Y_{n} \cong Q_{n}$.

\section{Conclusion}

In [14], the authors introduced the balanced hypercube to enhance some properties of the hypercube. Graph symmetry is an important factor in the design of an interconnection network. In 1997, it has been shown that the balanced hypercube is vertex-transitive. Recently, it was shown that the balanced hypercube is also arc-transitive. However, the full automorphism group of the balanced hypercube remained unknown. In this paper, we solve this problem. As applications, we first analyze the symmetry properties of the balanced hypercube and show that the balanced hypercube is not 2 -arc-transitive. Then, we give a theoretical explanation of the relationship between the balanced hypercube and the hypercube.

Acknowledgements: The authors are indebted to the anonymous referees for many valuable comments and constructive suggestions.

\section{References}

[1] N. Biggs, Algebraic graph theory, Cambridge university press, 1993.

[2] J. A. Bondy and U. S. R. Murty, Graph theory with applications, volume 290, Citeseer, 1976.

[3] H. Cheng and J.-X. Zhou, Edge neighbor connectivity of balanced hypercube, submitted.

[4] E. Dobson and J. Morris, Automorphism groups of wreath product digraphs., Electron. J. Comb. 16 (2009), research paper r17, 30.

[5] Y. Feng, Automorphism groups of Cayley graphs on symmetric groups with generating transposition sets., J. Comb. Theory, Ser. B 96 (2006), 67-72, doi:10.1016/j.jctb.2005.06.010.

[6] J. L. Gross and T. W. Tucker, Generating all graph coverings by permutation voltage assignments, Discrete Math. 18 (1977), 273-283.

[7] K. Huang and J. Wu, Balanced hypercubes., in: ICPP (1), 1992 pp. 80-84.

[8] K. Huang and J. Wu, Area efficient layout of balanced hypercubes, International Journal of High Speed Electronics and Systems 6 (1995), 631-645.

[9] K. Huang and J. Wu, Fault-tolerant resource placement in balanced hypercubes, Information Sciences 99 (1997), 159-172.

[10] Q.-X. Huang and Z. Zhang, On the Cayley graphs of $\operatorname{Sym}(n)$ with respect to transposition connection, submitted.

[11] P. Lorimer, Vertex-transitive graphs: Symmetric graphs of prime valency., J. Graph Theory 8 (1984), 55-68, doi:10.1002/jgt.3190080107.

[12] A. Malnič, Group actions, coverings and lifts of automorphisms., Discrete Math. 182 (1998), 203-218, doi:10.1016/S0012-365X(97)00141-6.

[13] G. Sabidussi, The composition of graphs, Duke Math. J 26 (1959), 693-696.

[14] J. Wu and K. Huang, The balanced hypercube: a cube-based system for fault-tolerant applications, IEEE Transactions on Computers 46 (1997), 484-490. 
[15] M. Xu, Automorphism groups and isomorphisms of Cayley digraphs., Discrete Math. 182 (1998), 309-319, doi:10.1016/S0012-365X(97)00152-0.

[16] M. Xu, X.-D. Hu and J.-M. Xu, Edge-pancyclicity and Hamiltonian laceability of the balanced hypercubes., Appl. Math. Comput. 189 (2007), 1393-1401, doi:10.1016/j.amc.2006.12.036.

[17] M.-C. Yang, Bipanconnectivity of balanced hypercubes., Comput. Math. Appl. 60 (2010), 1859-1867, doi:10.1016/j.camwa.2010.07.016.

[18] M.-C. Yang, Analysis of conditional diagnosability for balanced hypercubes, in: 2012 IEEE International Conference on Information Science and Technology, IEEE, 2012 pp. 651-654.

[19] M.-C. Yang, Super connectivity of balanced hypercubes., Appl. Math. Comput. 219 (2012), 970-975, doi:10.1016/j.amc.2012.06.077.

[20] Z. Zhang and Q. Huang, Automorphism group of bubble-sort graphs and modified bubble-sort graphs, Adv. Math. (China) 34 (2005), 441-447.

[21] J.-X. Zhou, The automorphism group of the alternating group graph, Applied Mathematics Letters 24 (2011), 229 - 231, doi:10.1016/j.aml.2010.09.009, http://www. sciencedirect.com/science/article/pii/s0893965910003319.

[22] J.-X. Zhou, Z.-L. Wu, S.-C. Yang and K.-W. Yuan, Symmetric property and reliability of balanced hypercube, IEEE Transactions on Computers 64 (2015), 876-881. 